\title{
A new and efficient method for $o$-Quinone methide intermediate generation: application to the biomimetic synthesis of $( \pm)$-Alboatrin.
}

\author{
Raphaël Rodriguez, Robert M. Adlington, John. E. Moses, Andrew cowley and \\ Jack E. Baldwin* \\ Chemistry Research Laboratory, University of Oxford, Mansfield Road, Oxford OXI \\ 3TA, United Kingdom
}

\section{Supporting informations}

\section{General experimental details:}

All solvents and reagents were purified by standard techniques reported in Perrin, D.D.; Amarego, W. L. F., Purification of Laboratory Chemicals, $3^{\text {rd }}$ edition, Pergamon Press, Oxford, 1988 or used as supplied from commercial sources as appropriate. Solvents were removed under reduced pressure using a Buchi R110 or R114 Rotavapor fitted with a water condenser. Final traces of solvent were removed from samples using an Edwards E2M5 high vacuum pump with pressures below $2 \mathrm{mmHg}$.

All experiments were carried out under inert atmosphere unless otherwise stated.

${ }^{1} \mathrm{H}$ NMR spectra were recorded at 200, 400 and $500 \mathrm{MHz}$ using, Bruker DPX 200, DQX400 and Bruker AMX500 instruments. For ${ }^{1} \mathrm{H}$ spectra recorded in $\mathrm{CDCl}_{3}$, $\mathrm{CD}_{3} \mathrm{OD}, \mathrm{C}_{6} \mathrm{D}_{6}$, chemical shifts are quoted in parts per million (ppm) and are referenced to the residual solvent peak. The following abbreviations are used: $\mathrm{s}$, singlet; d, doublet; $\mathrm{t}$, triplet; $\mathrm{m}$, multiplet; br, broad. Proton assignments and stereochemistry are supported by ${ }^{1} \mathrm{H}-{ }^{1} \mathrm{H}$ COSY and NOESY where necessary. Data are reported in the following manner: chemical shift (integration, multiplicity, coupling constant if appropriate). Coupling constants $(J)$ are reported in Hertz to the nearest $0.5 \mathrm{~Hz}$.

${ }^{13} \mathrm{C}$ NMR spectra were recorded at 50.2, 100.6 and 125.8 MHz using Bruker DPX200, Bruker DQX400, and Bruker AMX500 instruments. Carbon spectra assignments are supported by DEPT-135 spectra, ${ }^{13} \mathrm{C}-{ }^{1} \mathrm{H}$ (HMQC) correlations where necessary.

Chemical shifts are quoted in ppm and are referenced to the appropriate residual solvent peak.

Flash column chromatography was carried out using Sorbsil ${ }^{\mathrm{TM}}$ C60 (40-63 mm, 23040 mesh) silica gel. Thin layer chromatography was carried out on glass plates precoated with Merck silica gel $60 \mathrm{~F}_{254}$ which were visualised by quenching of u.v. fluorescence or by staining with $10 \% \mathrm{w} / \mathrm{v}$ ammonium molybdate in $2 \mathrm{M}$ sulphuric acid or $1 \% \mathrm{w} / \mathrm{v}$ potassium permanganate in aqueous alkaline solution followed by heat, as appropriate. 
Melting points were recorded using a Cambridge Instruments Gallen ${ }^{\mathrm{TM}}$ III Kofler Block melting apparatus or a Buchi 510 capillary apparatus and are uncorrected.

Infrared spectra were recorded either as a thin film between $\mathrm{NaCl}$ plates on a PerkinElmer Paragon 1000 Fourier Transform spectrometer with internal referencing. Absorption maxima are reported in wavenumbers $\left(\mathrm{cm}^{-1}\right)$.

Low resolution mass spectra were recorded on V. G. Micromass ZAB $1 \mathrm{~F}$ and V. G. Masslab instruments as appropriate with modes of ionisation being indicated as CI, EI, ES or APCI with only molecular ions. High resolution mass spectrometry was measured on a Waters 2790-Micromass LCT electrospray ionisation mass spectrometer and on a VG autospec chemical ionisation mass spectrometer.

\section{Experimental Procedures:}

\section{2-methyleneacetoxy-3-methyl-5-acetoxyphenol (6a)}<smiles>CC(=O)OCc1c(C)cc(OC(C)=O)cc1O</smiles>

To a stirred solution of 2,4-diacetoxy-6-methyl-benzaldehyde (5) (3.8 g, $16.1 \mathrm{mmol}$ ) in dry THF (90 ml) was slowly added a $2 \mathrm{M}$ THF solution of borane-DMS complex $(8.0 \mathrm{ml}, 16.1 \mathrm{mmol})$ at $0{ }^{\circ} \mathrm{C}$ under nitrogen. The reaction was stirred for one hour at room temperature and was then quenched at $0{ }^{\circ} \mathrm{C}$ with water $(2 \mathrm{ml})$. The mixture was evaporated to dryness under reduced pressure to give a crude which was purified by flash silica gel chromatography (70 : 30 30-40 P.E. : EtOAc). The title compound was obtained as a white solid which was crystallised in ether to afford (6a) as a white crystal $(3.2 \mathrm{~g}, 84 \%) . R_{\mathrm{F}} 0.3$ (70 : 30 30-40 P.E : EtOAc).

$\mathrm{Mp}=95-96^{\circ} \mathrm{C} ; v_{\max } / \mathrm{cm}^{-1}(\mathrm{NaCl}) 3413,1779,1735,1708,1599,1370,1209,1133 ; \delta_{\mathrm{H}}$ $\left(400 \mathrm{MHz}, \mathrm{CDCl}_{3}\right) 2.10(3 \mathrm{H}, \mathrm{s}), 2.27(3 \mathrm{H}, \mathrm{s}), 2.38(3 \mathrm{H}, \mathrm{s}), 5.12(2 \mathrm{H}, \mathrm{s}), 6.53(1 \mathrm{H}, \mathrm{s})$, $6.54(1 \mathrm{H}, \mathrm{s}) 8.23(1 \mathrm{H}, \mathrm{s}) ; \delta_{\mathrm{C}}\left(100 \mathrm{MHz}, \mathrm{CDCl}_{3}\right)$ 19.6, 21.0, 21.2, 59.6, 109.3, 115.8, 118.8, 141.2, 152.1, 157.3, 169.5, 174.3; HRMS (ES-) Calculated for $\mathrm{C}_{12} \mathrm{H}_{13} \mathrm{O}_{5}$ ([M $\left.\mathrm{H}^{-}\right)$: 237.0763, Found: 237.0763.

\section{( \pm )-Acetylalboatrin (7)}

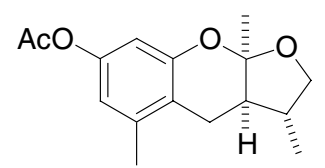

( \pm -Acetylalboatrin (7)

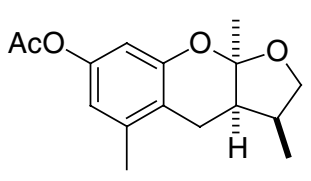

( \pm -Acetyl-epi-alboatrin<smiles>CC(=O)Oc1cc(C)c2c(c1)OC1(CC2)CC(C)CO1</smiles>

Spiroacetal (8)

In an sealed tube was stirred 2-methyleneacetoxy-3-methyl-5-acetoxyphenol (6a) (200 mg, $0.84 \mathrm{mmol}$ ) with ( \pm )-4,5-dihydro-2,4-dimethylfuran (3) (82 mg, $0.84 \mathrm{mmol})$ in benzene $(1 \mathrm{ml})$ at $80{ }^{\circ} \mathrm{C}$, under argon for 36 hours. After evaporation of benzene under reduced pressure, the colourless oil obtained was purified by flash silica gel 
chromatography (98 : 2 30-40 P.E. : EtOAc) to give a viscous colourless oil (57 mg, $25 \%)$ of spiroacetal (8) as an inseparable mixture [3:2] of two diastereoisomers $\left[R_{\mathrm{F}}\right.$ 0.5 (90 : 10 30-40 P.E : EtOAc)], and a white solid (158 mg, $68 \%$ ) of $( \pm)-$ acetylalboatrin (7) and ( \pm )-acetyl-epi-alboatrin as a mixture [12.6:1]. $R_{\mathrm{F}} 0.3$ (90: 10 30-40 P.E : EtOAc).

Data for $( \pm)-(7): v_{\max } / \mathrm{cm}^{-1}(\mathrm{NaCl}) 3054,2987,2305,1760,1603,1421,1262,1215$, 896,$752 ; \delta_{\mathrm{H}}\left(200 \mathrm{MHz}, \mathrm{CDCl}_{3}\right) 1.04(3 \mathrm{H}, \mathrm{d}, J 6.0 \mathrm{~Hz}), 1.50(3 \mathrm{H}, \mathrm{s}), 1.93(1 \mathrm{H}, \mathrm{ddd}, J$ 11.0, 4.5, 3.0 Hz), 2.12-2.17 (1H, m), $2.22(3 \mathrm{H}, \mathrm{s}), 2.26(3 \mathrm{H}, \mathrm{s}), 2.71-2.73(2 \mathrm{H}, \mathrm{m})$, $3.52(1 \mathrm{H}, \mathrm{t}, J 8.0 \mathrm{~Hz}), 4.17(1 \mathrm{H}, \mathrm{t}, J 8.0 \mathrm{~Hz}), 6.42(1 \mathrm{H}, \mathrm{d}, J 2.0 \mathrm{~Hz}), 6.49(1 \mathrm{H}, \mathrm{d}, J 2.0$ $\mathrm{Hz}) ; \delta_{\mathrm{C}}\left(100 \mathrm{MHz}, \mathrm{CDCl}_{3}\right) 15.9,19.5,21.2,22.0,22.8,35.4,47.9,74.1,107.1,108.3$, 114.9, 115.3, 138.1, 149.5, 153.6, 169.7; HRMS (ES+) Calculated for $\mathrm{C}_{16} \mathrm{H}_{21} \mathrm{O}_{4}([\mathrm{M}+$ $\left.\mathrm{H}]^{+}\right)$: 277.1440, Found: 277.1431.

\section{( \pm -Alboatrin (1)}

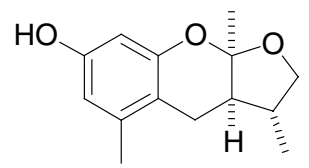

To a stirred solution of ( \pm )-acetylalboatrin (7) and ( \pm )-acetyl-epi-alboatrin [12.6:1] (480 mg, $1.74 \mathrm{mmol}$ ) in $9 \mathrm{ml}$ of $\mathrm{DCM} / \mathrm{MeOH} / \mathrm{H}_{2} \mathrm{O}(12 / 7 / 1)$ was quickly added potassium carbonate $(721 \mathrm{mg}, 5.21 \mathrm{mmol})$ at room temperature under nitrogen. The mixture was stirred for 6 hours and was then extracted with ethyl acetate $(3 \times 50 \mathrm{ml})$. The combined organic layers were washed with a saturated solution of ammonium chloride $(3 \times 50 \mathrm{ml})$, dried over magnesium sulphate and concentrated under reduced pressure. The crude obtained was purified by flash silica gel chromatography ( $98: 2$ 30-40 P.E. : EtOAc) to give a white solid. The solid was crystallised in ether to afford ( \pm )-Alboatrin (1) $(368 \mathrm{mg}, 90 \%)$ as white crystals. $R_{\mathrm{F}} 0.1$ (90 : 10 30-40 P.E : EtOAc).

$\mathrm{Mp}=148-149{ }^{\circ} \mathrm{C} ; v_{\max } / \mathrm{cm}^{-1}(\mathrm{NaCl}) 3400,2958,1620,1599,1494,1462,1335,1204$, 1148, 1117, 986, 845; $\delta_{\mathrm{H}}\left(200 \mathrm{MHz}, \mathrm{CDCl}_{3}\right) 1.04(3 \mathrm{H}, \mathrm{d}, J 6.5 \mathrm{~Hz}), 1.51(3 \mathrm{H}, \mathrm{s}), 1.93$ (1H, ddd, $J 11.0,4.5,3.5 \mathrm{~Hz}), 2.01-2.14(1 \mathrm{H}, \mathrm{m}), 2.19(3 \mathrm{H}, \mathrm{s}), 2.68(2 \mathrm{H}, \mathrm{br} \mathrm{s}), 3.51$ $(1 \mathrm{H}, \mathrm{t}, J 8.5 \mathrm{~Hz}), 4.17(1 \mathrm{H}, \mathrm{t}, J 8.5 \mathrm{~Hz}), 4.80(1 \mathrm{H}, \mathrm{br} \mathrm{s}), 6.26(1 \mathrm{H}, \mathrm{s}), 6.29(1 \mathrm{H}, \mathrm{s}) ; \delta_{\mathrm{C}}$ $\left(100 \mathrm{MHz}, \mathrm{CDCl}_{3}\right)$ 16.0, 19.4, 21.5, 23.2, 35.5, 48.4, 74.0, 101.9, 107.5, 109.6, 110.0, 138.2, 153.7, 155.1; HRMS (ES-) Calculated for $\mathrm{C}_{14} \mathrm{H}_{17} \mathrm{O}_{3}\left([\mathrm{M}-\mathrm{H}]^{-}\right)$: 233.1178, Found: 233.1180. 


\section{Compound (9)}

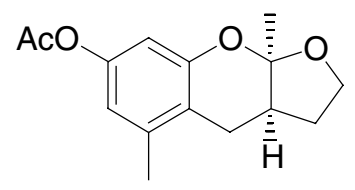

In a sealed tube was stirred 2-methyleneacetoxy-3-methyl-5-acetoxyphenol (6a) (203 $\mathrm{mg}, 0.85 \mathrm{mmol})$ in 4,5 -dihydro-2-methylfuran $(1.0 \mathrm{ml}, 10.9 \mathrm{mmol})$ at $100{ }^{\circ} \mathrm{C}$, under argon for 12 hours. After evaporation of excess of reagent under reduced pressure, the yellow oil obtained was purified by flash silica gel chromatography $(95: 5$ 30-40 P.E. : EtOAc) to give a white solid $(175 \mathrm{mg}, 78 \%) . \mathrm{Mp}=115-117^{\circ} \mathrm{C} ; R_{\mathrm{F}} 0.2(90: 1030$ 40 P.E : EtOAc); $v_{\max } / \mathrm{cm}^{-1}$ (NaCl) 3056, 2982, 1757, 1597, 1482, 1368, 1265, 1213 , 1109,$736 ; \delta_{\mathrm{H}}\left(400 \mathrm{MHz}, \mathrm{CDCl}_{3}\right) 1.51(3 \mathrm{H}, \mathrm{s}), 1.75-1.85(1 \mathrm{H}, \mathrm{m}), 2.01-2.09(1 \mathrm{H}, \mathrm{m})$, $2.21(3 \mathrm{H}, \mathrm{s}), 2.26(3 \mathrm{H}, \mathrm{s}), 2.43-2.49(1 \mathrm{H}, \mathrm{m}), 2.78(1 \mathrm{H}, \mathrm{s}), 2.79(1 \mathrm{H}, \mathrm{s}), 3.95(1 \mathrm{H}, \mathrm{dd}$, $J 16.5,8.5 \mathrm{~Hz}), 4.03(1 \mathrm{H}, \mathrm{td}, J 8.5,3.0 \mathrm{~Hz}), 6.43\left(1 \mathrm{H}\right.$, br s), $6.50(1 \mathrm{H}, \mathrm{br} \mathrm{s}) ; \delta_{\mathrm{C}}(100$ $\left.\mathrm{MHz}, \mathrm{CDCl}_{3}\right)$ 19.4, 21.2, 22.4, 23.5, 29.0, 40.5, 66.7, 106.3, 108.2, 115.0, 115.3, 138.1, 149.5, 153.8, 169.7; HRMS (ES+) Calculated for $\mathrm{C}_{15} \mathrm{H}_{19} \mathrm{O}_{4}\left([\mathrm{M}+\mathrm{H}]^{+}\right)$: 263.1283, Found: 263.1289.

\section{Compound (10)}

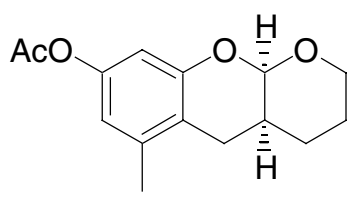

In a sealed tube was stirred 2-methyleneacetoxy-3-methyl-5-acetoxyphenol (6a) (203 $\mathrm{mg}, 0.85 \mathrm{mmol})$ in 3,4-dihydro- $2 \mathrm{H}$-pyran $(1.0 \mathrm{ml}, 10.9 \mathrm{mmol})$ at $100{ }^{\circ} \mathrm{C}$, under argon for 12 hours. After evaporation of excess of reagent under reduced pressure, the yellow oil obtained was purified by flash silica gel chromatography ( $95: 530-40$ P.E. : EtOAc) to give a colourless oil (161 mg, $72 \%) . R_{\mathrm{F}} 0.3$ (90 : 10 30-40 P.E : EtOAc); $v_{\max } / \mathrm{cm}^{-1}(\mathrm{NaCl}) 3055,2934,1759,1598,1422,1265,1217,1093,897,739 ; \delta_{\mathrm{H}}(400$ $\left.\mathrm{MHz}, \mathrm{CDCl}_{3}\right)$ 1.62-1.70 (5H, m), $2.18(3 \mathrm{H}, \mathrm{s}), 2.26(3 \mathrm{H}, \mathrm{s}), 2.50(1 \mathrm{H}, \mathrm{dd}, J 16.5,4.5$ $\mathrm{Hz}), 2.72(1 \mathrm{H}, \mathrm{dd}, J 16.5,6.5 \mathrm{~Hz}), 3.69-3.73(1 \mathrm{H}, \mathrm{m}), 3.98-4.04(1 \mathrm{H}, \mathrm{m}), 5.28(1 \mathrm{H}, \mathrm{d}$, $J 2.5 \mathrm{~Hz}), 6.49(1 \mathrm{H}, \mathrm{s}), 6.50(1 \mathrm{H}, \mathrm{s}) ; \delta_{\mathrm{C}}\left(100 \mathrm{MHz}, \mathrm{CDCl}_{3}\right)$ 19.4, 21.2, 23.6, 24.4, 26.5, 31.6, 62.7 , 96.3, 107.8, 115.6, 116.4, 138.3, 149.4, 153.3, 169.7; HRMS (CI+) Calculated for $\mathrm{C}_{15} \mathrm{H}_{19} \mathrm{O}_{4}\left([\mathrm{M}+\mathrm{H}]^{+}\right)$: 263.1283, Found: 263.1288 . 


\section{Compound (11)}

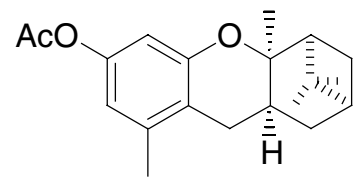

In a sealed tube was stirred 2-methyleneacetoxy-3-methyl-5-acetoxyphenol (6a) (203 $\mathrm{mg}, 0.85 \mathrm{mmol})$ in $(1 \mathrm{R})-(+)-\alpha$-pinene $(1.0 \mathrm{ml}, 6.3 \mathrm{mmol})$ at $140{ }^{\circ} \mathrm{C}$, under argon for 12 hours. After evaporation of excess of pinene under reduced pressure, the yellow oil obtained was purified by flash silica gel chromatography (99: $130-40$ P.E. : EtOAc) to give a viscous colourless oil $(81 \mathrm{mg}, 30 \%)$. $[\alpha]_{\mathrm{D}}^{25}=-2.9\left(c=1, \mathrm{CHCl}_{3}\right) ; R_{\mathrm{F}} 0.6(98$ : 2 30-40 P.E : EtOAc); $v_{\max } / \mathrm{cm}^{-1}(\mathrm{NaCl}) 2922,1758,1559,1483,1369,1216,1127$, 1015,$739 ; \delta_{\mathrm{H}}\left(500 \mathrm{MHz}, \mathrm{CDCl}_{3}\right) 1.04(3 \mathrm{H}, \mathrm{s}), 1.16(1 \mathrm{H}, \mathrm{d}, J 10.5 \mathrm{~Hz}), 1.28(3 \mathrm{H}, \mathrm{s})$, $1.31(3 \mathrm{H}, \mathrm{s}), 1.32-1.35(1 \mathrm{H}, \mathrm{m}), 1.81-1.84(1 \mathrm{H}, \mathrm{m}), 2.04-2.15(2 \mathrm{H}, \mathrm{m}), 2.17(1 \mathrm{H}, \mathrm{t}, J$ $5.5 \mathrm{~Hz}), 2.24(3 \mathrm{H}, \mathrm{s}), 2.25(3 \mathrm{H}, \mathrm{s}), 2.43(1 \mathrm{H}, \mathrm{dd}, J 15.0,5.0 \mathrm{~Hz}), 2.47-2.53(1 \mathrm{H}, \mathrm{m})$, $2.73(1 \mathrm{H}, \mathrm{dd}, J 15.0,6.0 \mathrm{~Hz}), 6.40(1 \mathrm{H}, \mathrm{d}, J 2.5 \mathrm{~Hz}), 6.49(1 \mathrm{H}, \mathrm{d}, J 2.5 \mathrm{~Hz}) ; \delta_{\mathrm{C}}(125$ $\left.\mathrm{MHz}, \mathrm{CDCl}_{3}\right)$ 19.2, 21.2, 23.2, 26.8, 27.5, 28.0, 29.3, 34.2, 34.5, 40.2, 40.4, 54.9, 84.0, 108.3, 114.9, 121.3, 136.8, 149.3, 157.3, 169.7; HRMS (ES+) Calculated for $\mathrm{C}_{20} \mathrm{H}_{27} \mathrm{O}_{3}\left([\mathrm{M}+\mathrm{H}]^{+}\right): 315.1960$, Found: 315.1963 .

\section{Compound (12)}

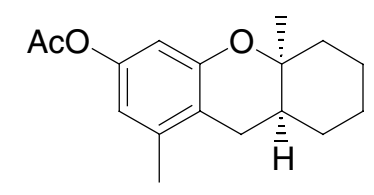

In a sealed tube was stirred 2-methyleneacetoxy-3-methyl-5-acetoxyphenol (6a) (203 $\mathrm{mg}, 0.85 \mathrm{mmol})$ in 1 -methylcyclohexene $(1.0 \mathrm{ml}, 8.5 \mathrm{mmol})$ at $140{ }^{\circ} \mathrm{C}$, under argon for 12 hours. After evaporation of excess of methylcyclohexene under reduced pressure, the yellow oil was purified by flash silica gel chromatography $(99: 130-40$ P.E. : EtOAc) to give a white solid (141 mg, $60 \%)$. Mp $=100-101{ }^{\circ} \mathrm{C} ; R_{\mathrm{F}} 0.6(98: 2$ 30-40 P.E : EtOAc); $v_{\max } / \mathrm{cm}^{-1}(\mathrm{NaCl}) 2933,1758,1596,1370,1265,1218,1125,737$; $\delta_{\mathrm{H}}\left(400 \mathrm{MHz}, \mathrm{CDCl}_{3}\right) 1.17(3 \mathrm{H}, \mathrm{s}), 1.24-1.31(2 \mathrm{H}, \mathrm{m}), 1.36-1.44(2 \mathrm{H}, \mathrm{m}), 1.48-1.52$ $(1 \mathrm{H}, \mathrm{m}), 1.57-1.75(3 \mathrm{H}, \mathrm{m}), 1.93(1 \mathrm{H}, \mathrm{bd}, J 13.5 \mathrm{~Hz}), 2.18(3 \mathrm{H}, \mathrm{s}), 2.24(1 \mathrm{H}, \mathrm{d}, J 17.0$ $\mathrm{Hz}), 2.25(3 \mathrm{H}, \mathrm{s}), 2.81(1 \mathrm{H}, \mathrm{dd}, J 17.0,6.5 \mathrm{~Hz}), 6.40(1 \mathrm{H}, \mathrm{d}, J 2.0 \mathrm{~Hz}), 6.44(1 \mathrm{H}, \mathrm{d}, J$ $2.0 \mathrm{~Hz}) ; \delta_{\mathrm{C}}\left(100 \mathrm{MHz}, \mathrm{CDCl}_{3}\right) 19.4,21.2,21.7,25.4,25.6,27.1,28.8,36.8,38.3$, 74.7, 108.0, 114.3, 116.4, 138.4, 149.1, 153.8, 169.8; HRMS (CI+) Calculated for $\mathrm{C}_{17} \mathrm{H}_{23} \mathrm{O}_{3}\left([\mathrm{M}+\mathrm{H}]^{+}\right): 275.1647$, Found: 275.1644 . 


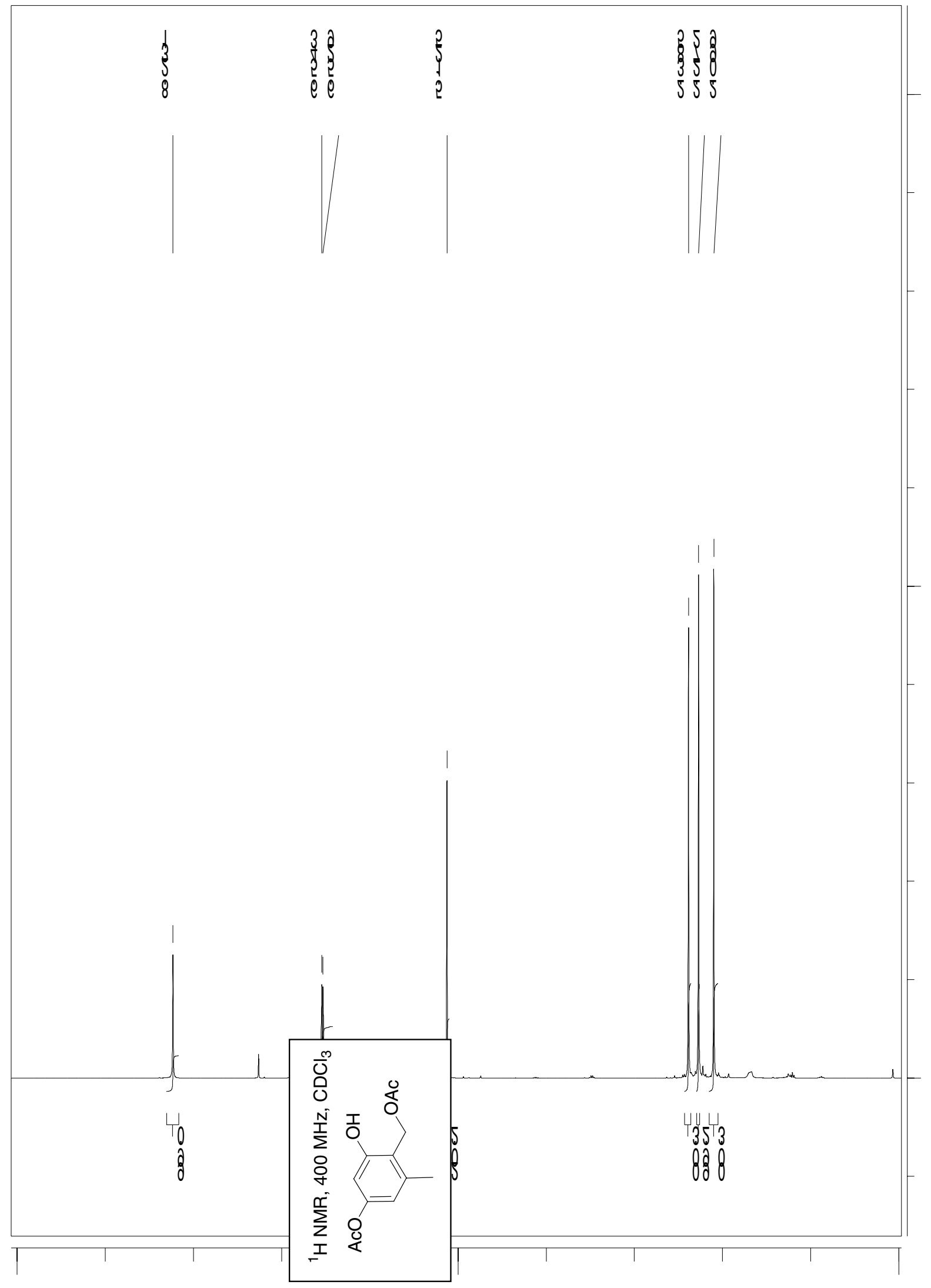




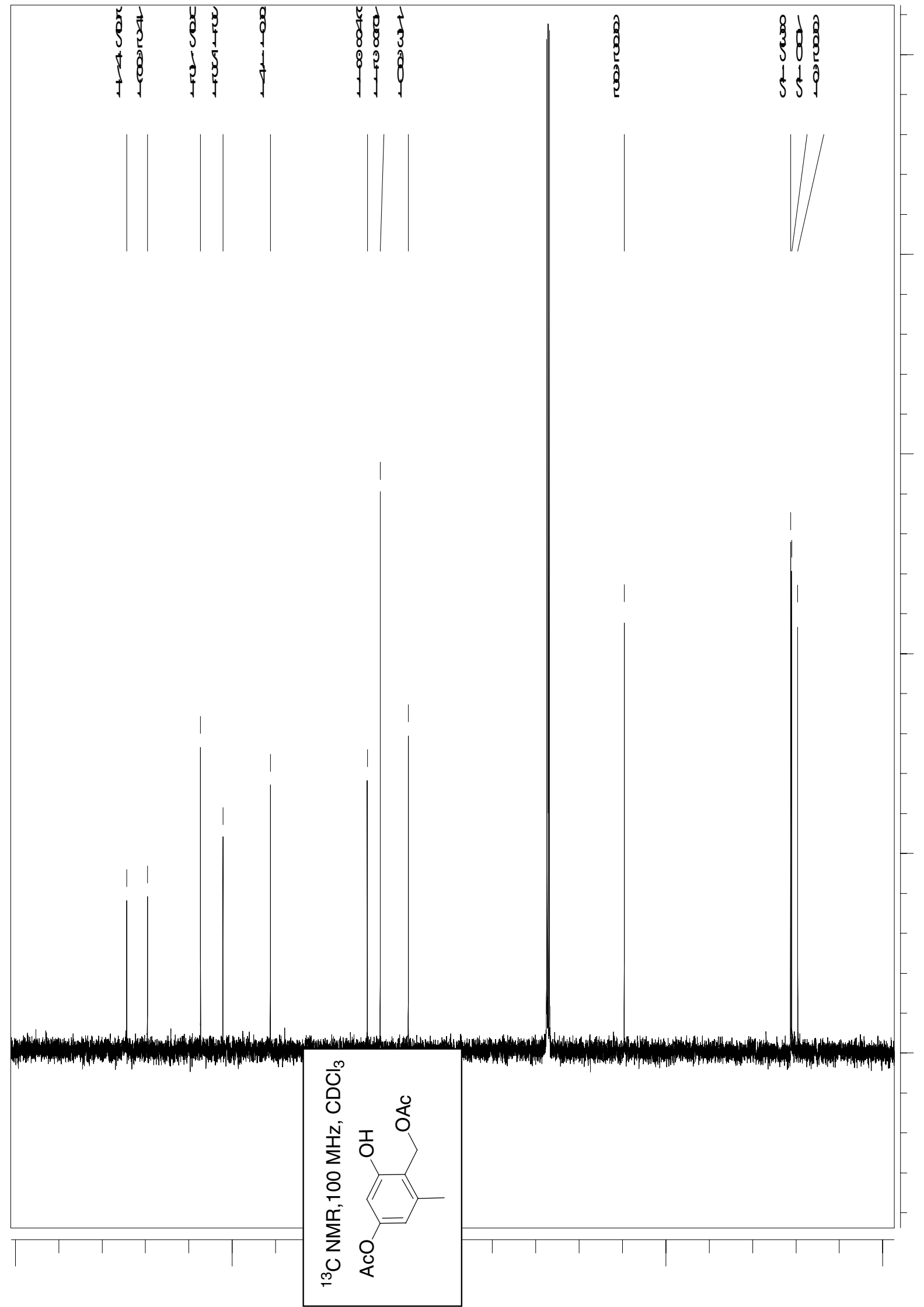




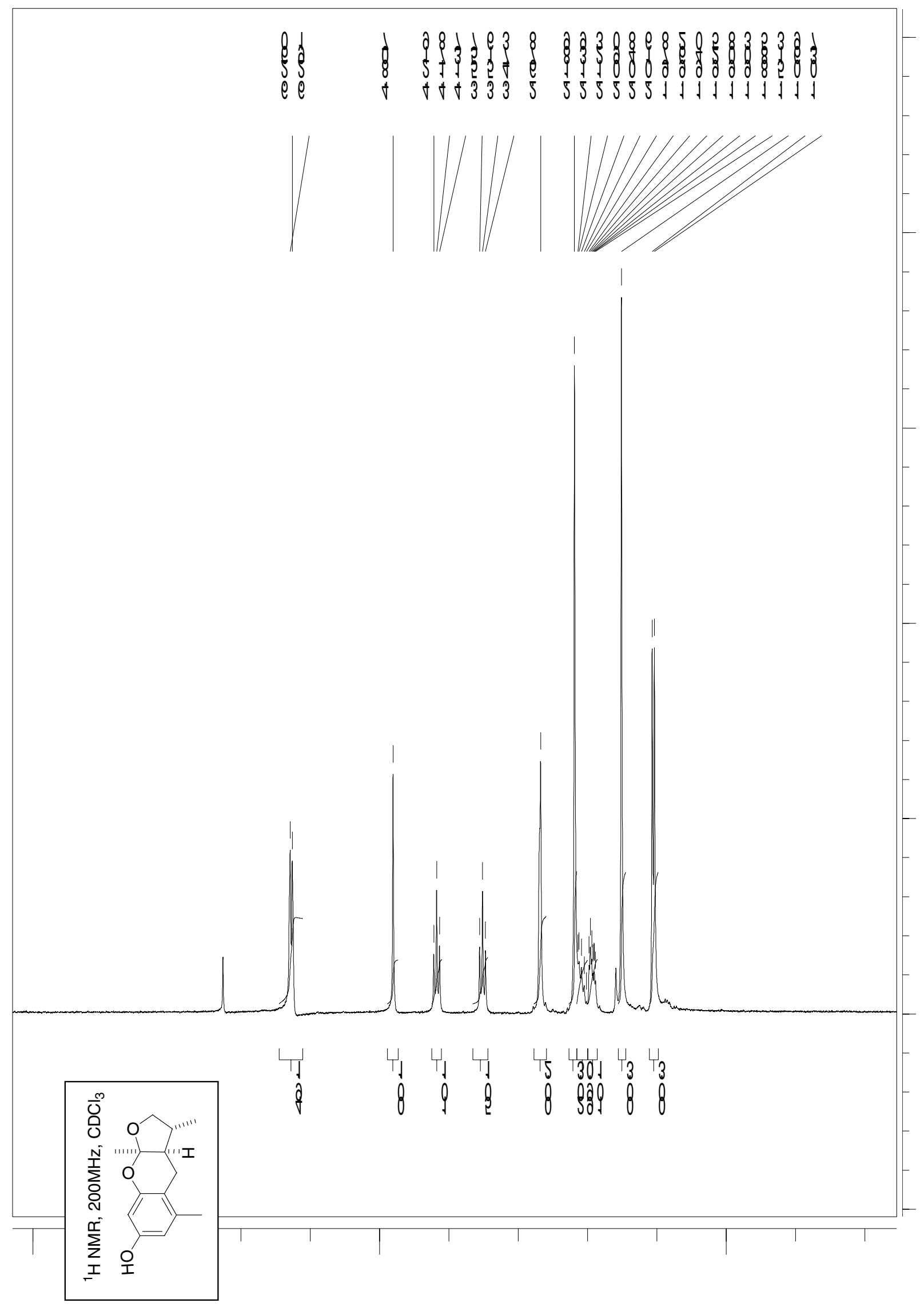




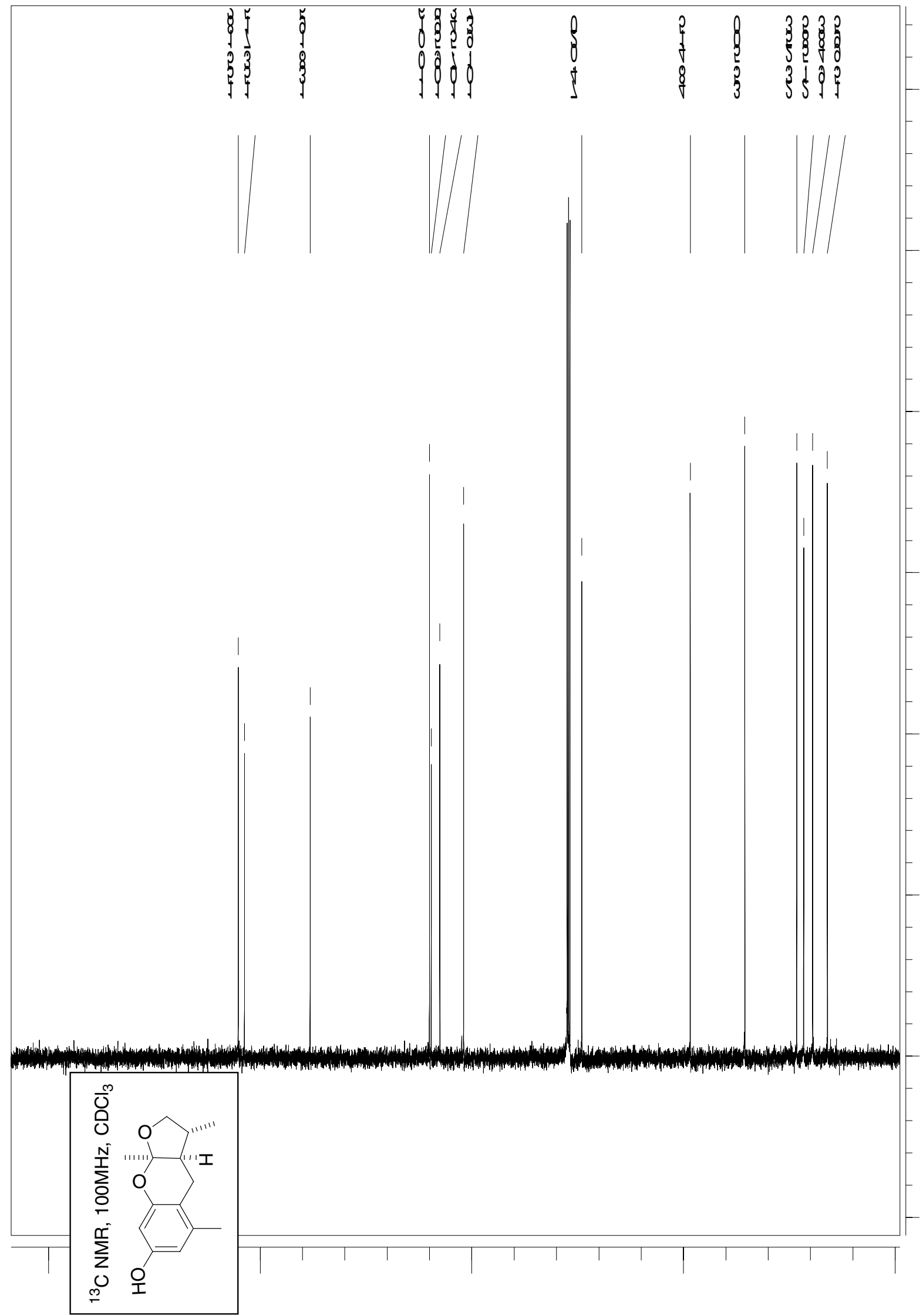




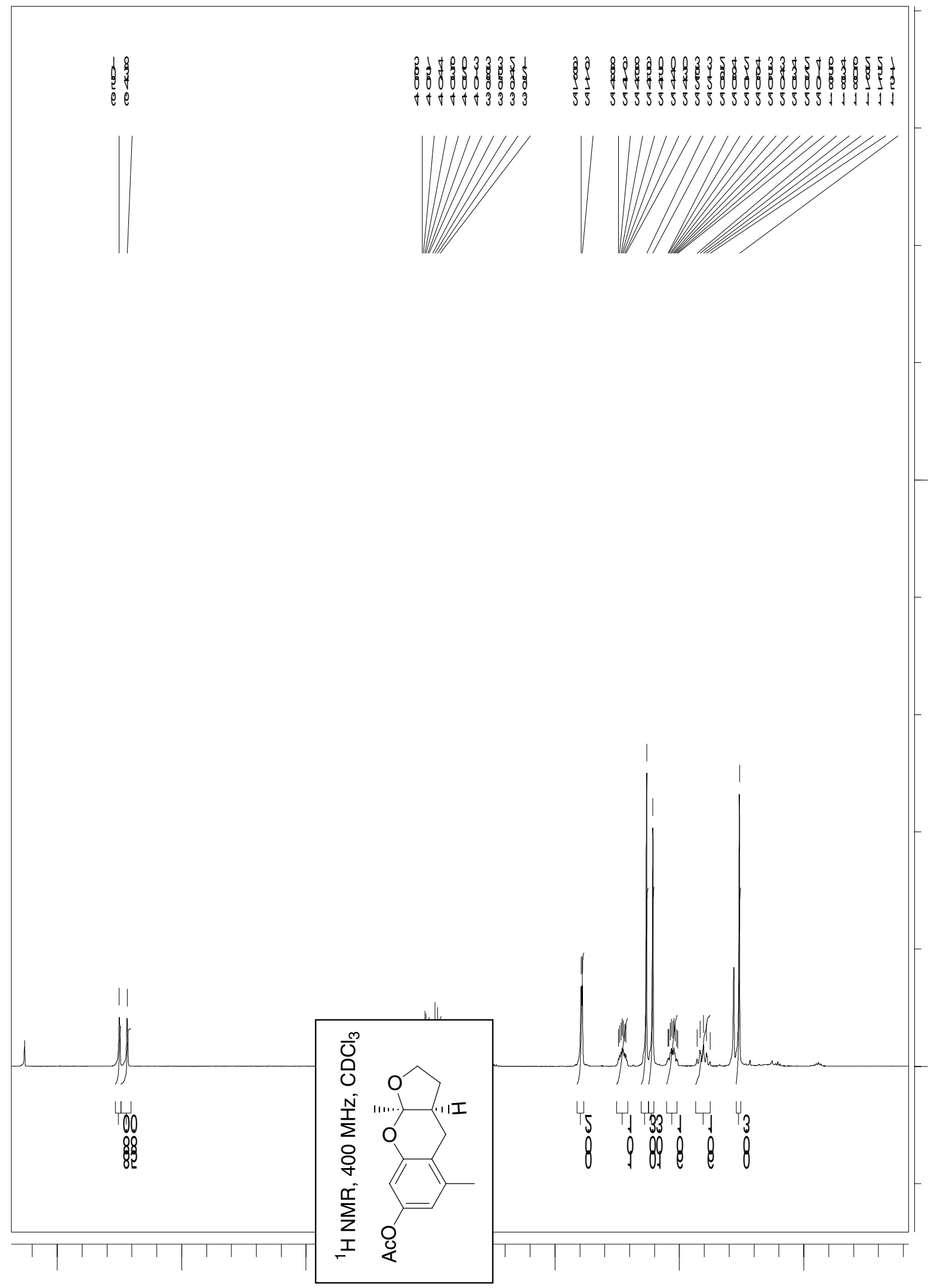




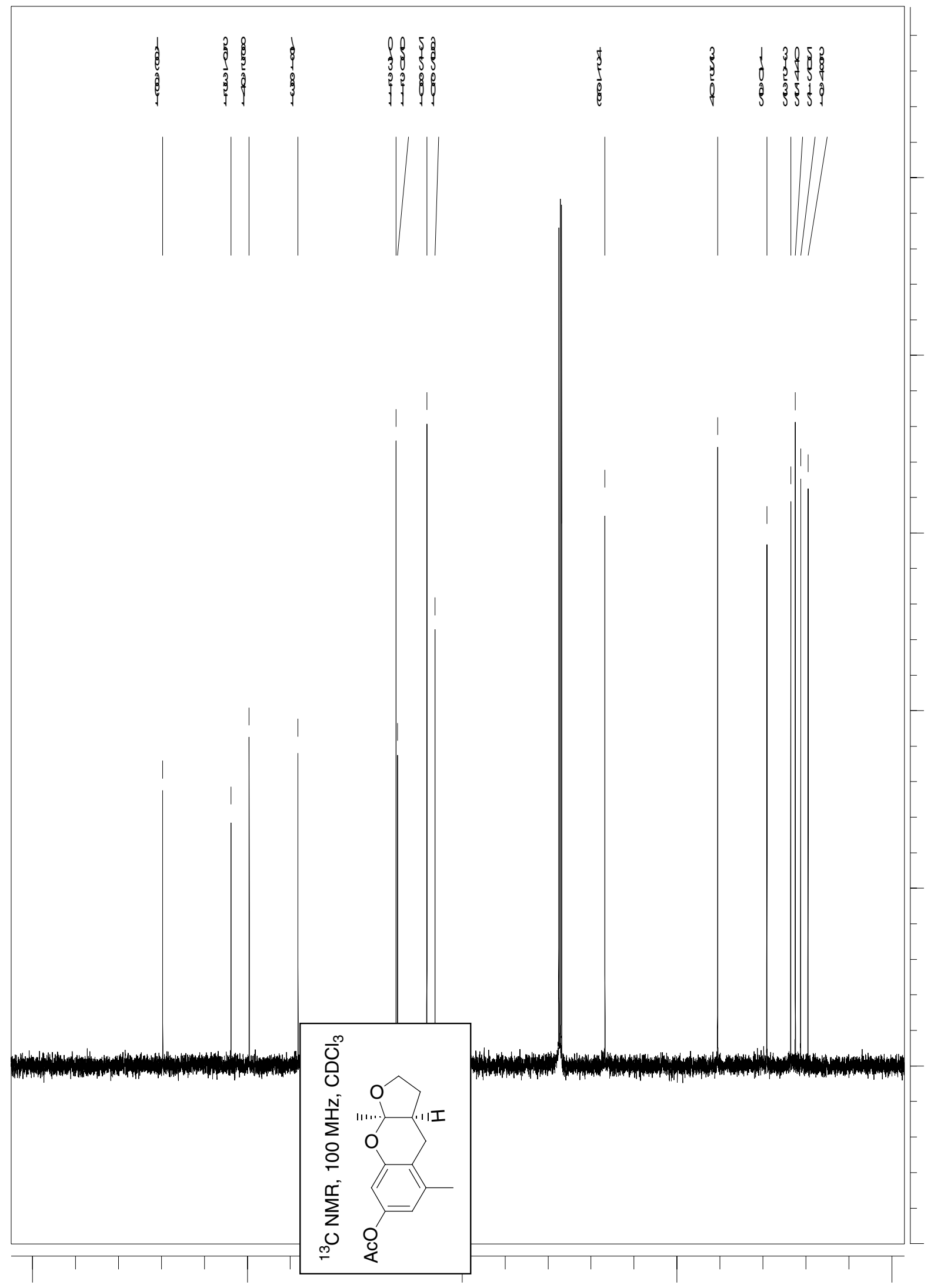




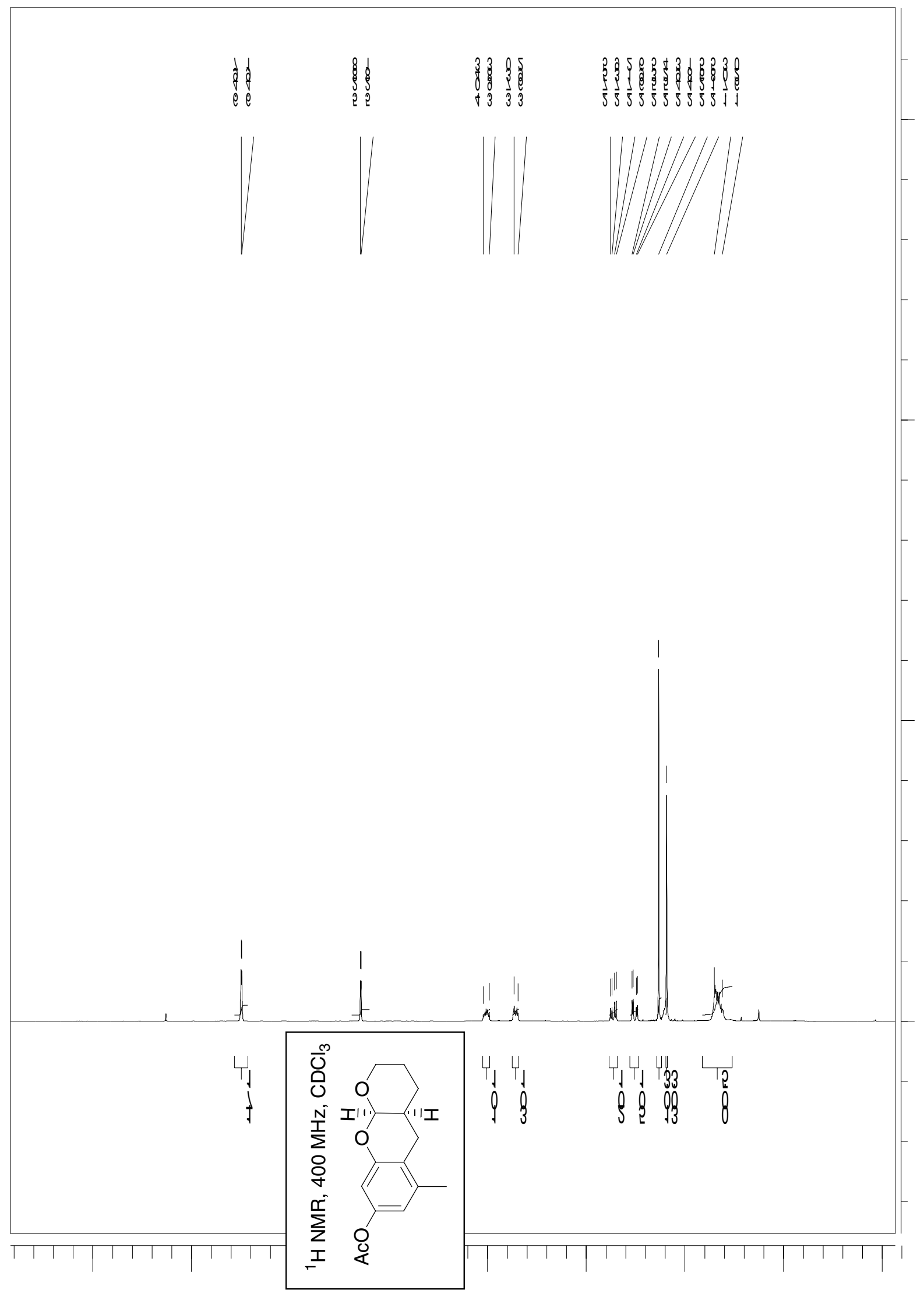




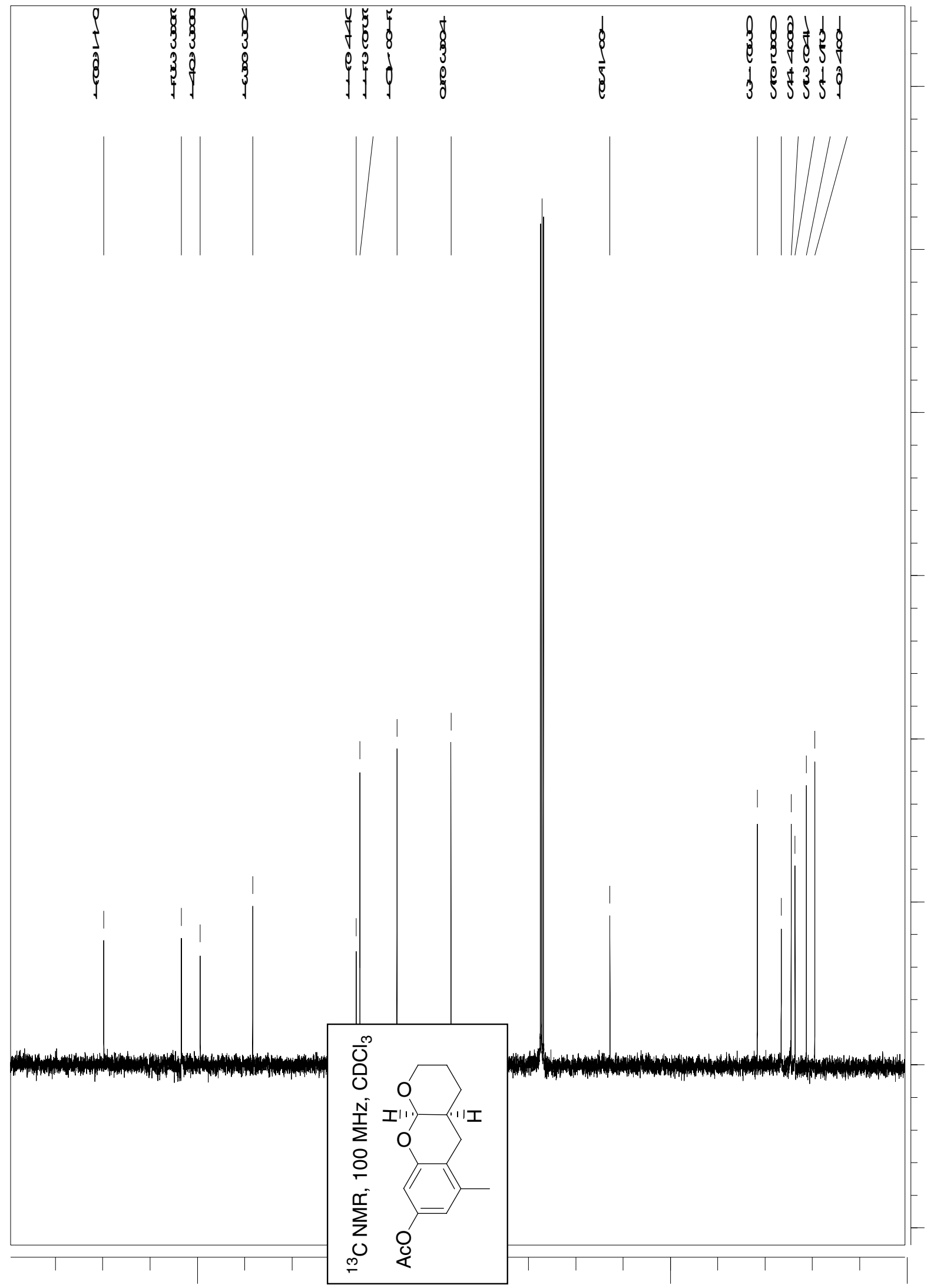




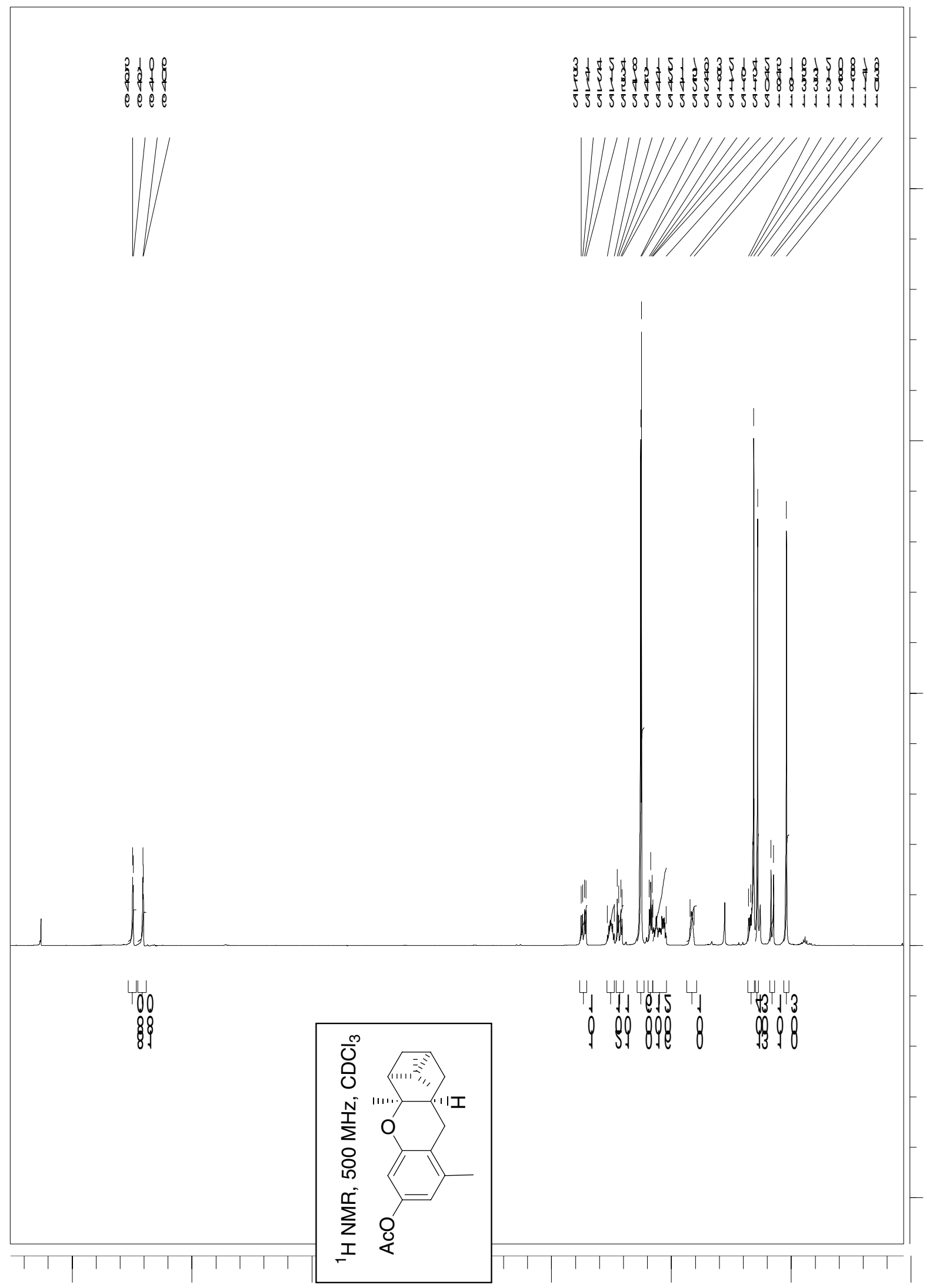




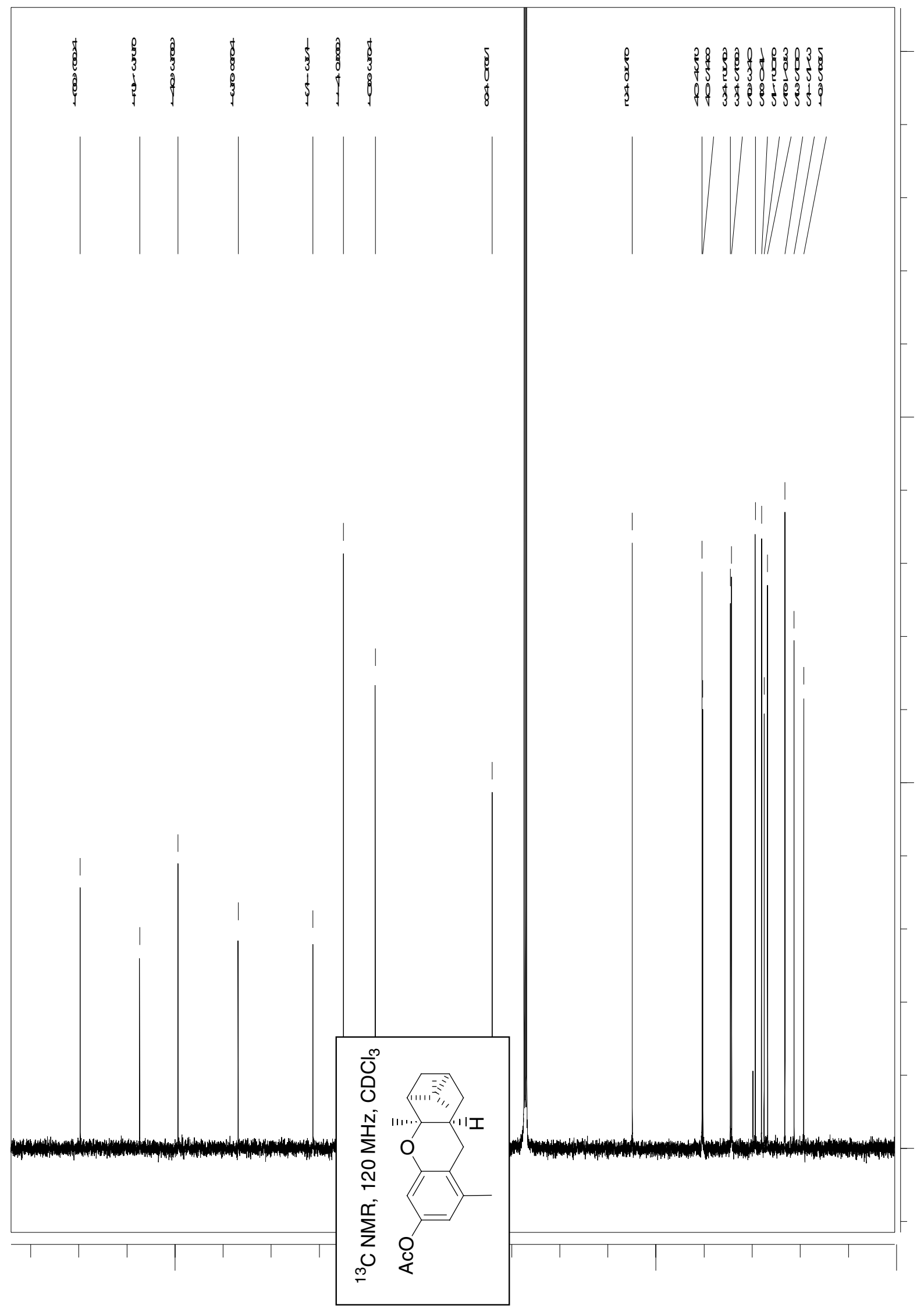




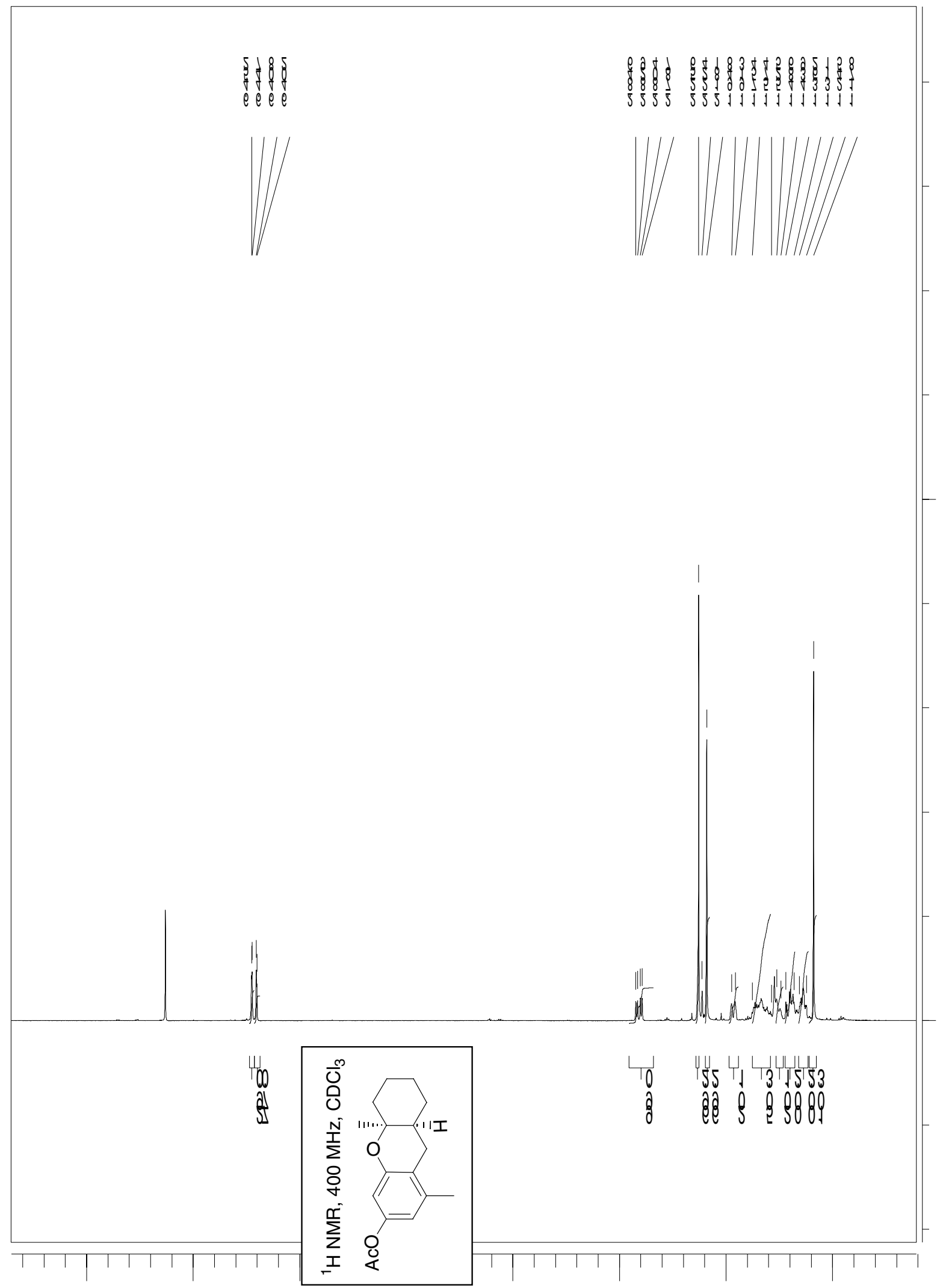




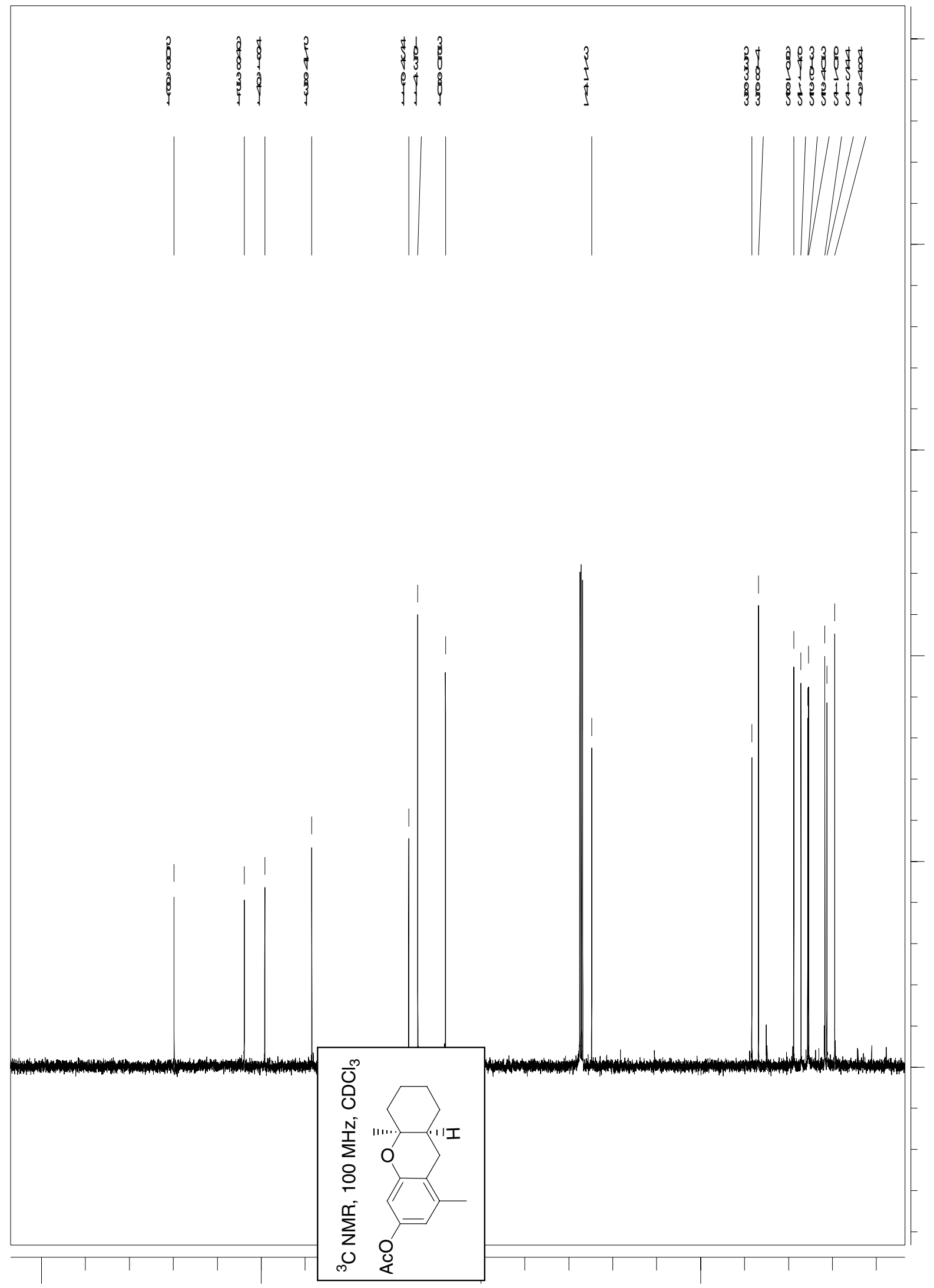


